Hariyan S. V. Morphological rearrangement of large joints of the lower extremities under the conditions of a combination of abdominal and orthopedic trauma complicated by ischemia-reperfusion of the extremity. Journal of Education, Health and Sport. 2020;10(4):297-305. eISSN 2391-8306. DOI http://dx.doi.org/10.12775/JEHS.2020.10.04.033

https://apcz.umk.pl/czasopisma/index.php/JEHS/article/view/JEHS.2020.10.04.033

https://zenodo.org/record/4097786

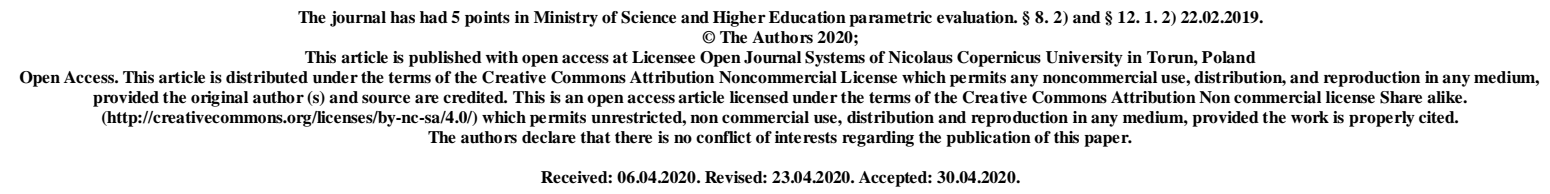

UDK 616.728-091.8-02:617.54/55-001-06:616.137-005.4-007.271

\title{
MORPHOLOGICAL REARRANGEMENT OF LARGE JOINTS OF THE LOWER EXTREMITIES UNDER THE CONDITIONS OF A COMBINATION OF ABDOMINAL AND ORTHOPEDIC TRAUMA COMPLICATED BY ISCHEMIA- REPERFUSION OF THE EXTREMITY
}

\author{
S. V. Hariyan \\ State Organization "Ukrainian Scientific and Practical Center of Emergency and \\ Disaster Medicine Ministry of Health of Ukraine", Kyiv, Ukraine
}

\section{Abstract}

Introduction. Injuries are among the pressing problems of modern urban society. The structure of injuries is dominated by multiple and combined injuries, which are characterized by significant severity and high mortality and lead to multiple organ dysfunction and insufficiency. In recent years, studies of the functional state of the liver have been widely used as a model for the development of multiple organ dysfunction in conditions of severe experimental trauma.

The objective is to determine the nature of disorders of biliary function of the liver in conditions of combined trauma to the abdomen and chest of rats in the early period of traumatic disease and to evaluate the effectiveness of their correction with thiotriazoline.

Research materials and their discussion. The experiments were performed on 86 nonlinear white male rats weighing 200-220 g. All animals were divided into five groups i.e. one control group and four experimental ones. Injuries were performed under thiopental- 
sodium anesthesia. The control group included intact animals that had just been anesthetized. In the first experimental group, the animals were simulated chest trauma. In the second experimental group, a blunt trauma was simulated. In the third experimental group, these injuries were combined. In the fourth experimental group, animals with combined trauma were injected intraperitoneally with thiotriazoline at a dose of $9.1 \mathrm{mg}-\mathrm{kg}^{-1} .1,3$ and 7 days after injury under thiopentalone sodium anesthesia in animals, the common bile duct was catheterized and bile was collected for $60 \mathrm{~min}$. It was set the rate of bile excretion. The concentration of total bile acids and conjugated bilirubin was determined in bile, and the rate of their excretion was calculated.

Research results and their discussion. Under conditions of isolated trauma of the chest, abdomen and their combination, there is a significant violation of biliary function of the liver, which is primarily manifested by a decrease in the rate of bile secretion and excretion of its main components - cholates and conjugated bilirubin. According to the severity of hepatic dysfunction, they can be divided as follows: isolated chest injury $\leftarrow$ isolated abdominal injury $\leftarrow$ combined injury. Under conditions of isolated chest trauma, the studied parameters in comparison with the control reach a minimum level of up to 3 days and normalize up to 7 days. After applying an isolated abdominal injury, the rate of bile secretion and the rate of excretion of total bile acids are reduced after 1 day. However, all the studied indicators reach a minimum value after 3 days and remain at the same level until 7 days. After a combined injury, the rates gradually decrease to 7 days and in each subsequent period become significantly lower than in the previous one. The use for the correction of detected disorders of thiotriazoline in the group of animals with combined trauma compared with animals without correction contributed to a significant increase in the rate of bile excretion, excretion of total bile acids and conjugated bilirubin. Although up to 7 days the indicators did not reach the level of the control group, it can be argued that thiotriazoline has a positive effect on biliary function of the liver.

Conclusion. Combined trauma to the abdomen and chest contributes to a greater decrease in liver function, compared with isolated lesions. They are based on the suppression of the rate of bile secretion and excretion of total bile acids and conjugated bilirubin. The use of thiotriazoline at a dose of $9.1 \mathrm{mg} \square \mathrm{kg}-1$ in animals with combined abdominal trauma is accompanied by a significant decrease in biliary dysfunction.

Key words: combined trauma; chest; abdomen; bile secretion; thiotriazoline. 
Problem statement and analysis of recent research and publications. Combined trauma remains the leading cause of death and injury among people of working age. The number of injured is suposed to be increasing over the next 20 years [1].

Clinical and experimental studies have shown that combined trauma is associated with significantly longer fracture healing times and a higher incidence of complications compared to isolated fractures. In addition, it was determined that the local and systemic inflammatory response significantly affects the healing process of fractures [2-4].

One of the most common complications and the cause of disability, the frequency of which is constantly increasing, is osteoarthritis. Trauma, including joints, is an important risk factor for post-traumatic osteoarthritis. Scientists have currently clearly proven the negative impact of systemic inflammatory reactions on the development of post-traumatic osteoarthritis [5, 6]. However, these studies mainly concern local injuries.

The basis of joint damage due to traumatic injuries are changes in cartilage. The pathophysiology of the process is the degeneration and death of cartilage cells, depolymerization of the main substance produced by chondrocytes develops, the amount of glycosaminoglycans decreases, which leads to progressive cartilage degeneration. There is growth of bone tissue and the formation of osteophytes. The synovial membrane, fibrous capsule and periarticular muscles are involved in the pathological process. According to statistics, the first place in the frequency of lesions is occupied by the hip joint, the second the knee, the third - the shoulder [7,8].

The purpose of the work is to investigate the features of morphological disorders of the tissues of the large joints of the lower extremities in the conditions of simulated injuries at a later date.

Materials and methods. The working hypothesis of the experimental study is the assumption that the use of a tourniquet and subsequent reperfusion of ischemic tissues leads to significant systemic changes with subsequent dysfunction of large joints in the late posttraumatic period with combined abdominal and skeletal trauma on the background of hypovolemic shock.

In order to achieve this goal on 40 nonlinear male rats weighing 190-220g, an experimental study has been performed.

All injury experiments were made under thiopental-sodium anesthesia (40 mg • kg-1 mass). Closed fracture of the femur was modeled by applying a single dosed blow to a specially designed device on the thigh, which caused a closed fracture - the plane of the impact device was $0.5 \mathrm{~cm}^{2}$, the impact force is more than $120 \mathrm{~kg} / \mathrm{cm}^{2}$. Closed trauma of the 
abdominal cavity was modeled by applying two dosed blows to the abdominal cavity by a specially designed device. Due to the dosed blow to the abdominal cavity, a closed trauma of the abdominal organs is simulated. The impact force of the device is calculated in such a way as to cause a closed injury of the abdominal organs without massive blood loss - the plane of the impact device was $2.5 \mathrm{~cm}^{2}$, the impact force is not more than $60 \mathrm{~kg} / \mathrm{cm}^{2}$. At such parameters there were intraorganic hematomas with damage to the parenchyma and small intra-organ blood vessels, there were also bruises and small radial ruptures of parenchymal organs.

Massive external blood loss was modeled by performing sufficient surgical access to the femoral vessels on the opposite side from the fracture of the femur, then a ligature was inserted under the vascular bundle and the vessels are dissected. After that $20-22 \%$ of circulating blood was taken for 1 min (acute blood loss). Blood was collected by soaking and weighing a cotton swab. Immediately after taking enough blood, the bleeding was stopped by tying ligatures.

A modification of a wide elastic hemostatic tourniquet, trademark "SWAT®", made in the USA, was used as hemostatic tourniquets. Prior to application, a piece $1.5 \mathrm{~cm}$ wide and 10 $\mathrm{cm}$ long was cut from the original hemostatic tourniquet cloth. Sufficient stretching of the tourniquet, for complete compression of the vessels of the lower paw of animals, was controlled by special indicators applied by the manufacturer to its surface.

Animals were removed from the experiment 3, 7, 14 and 21 days after simulation of injuries. Under thiopental-sodium anesthesia, all rats of the corresponding group were slaughtered by total bloodletting from the heart. Histological changes in the tissues of the hip and knee joints were studied after 21 days of the post-traumatic period. Tissue samples of the hip and knee joints were taken for histological examination. Sections 5-6 $\mathrm{mm}$ thick, stained with hematoxylin and eosin, were examined and documented using a microscope LOMO Biolam I.

The study of these indicators was determined in the Central Scientific Laboratory of Ternopil State Medical University named after I. Ya. Gorbachevsky.

Research results. Microscopic studies of the hip joint of animals with experimental trauma showed that in the superficial hyaline cartilaginous plate there were areas with a violation of the ordered arrangement of chondrocytes. In some chondrocytes of the first and second orders the nuclei are changed, their pyknosis is present. Areas of cartilage are noted, where there are no cells, and in their place there are moderately basophilic areas of intercellular substance (Fig. 1). 


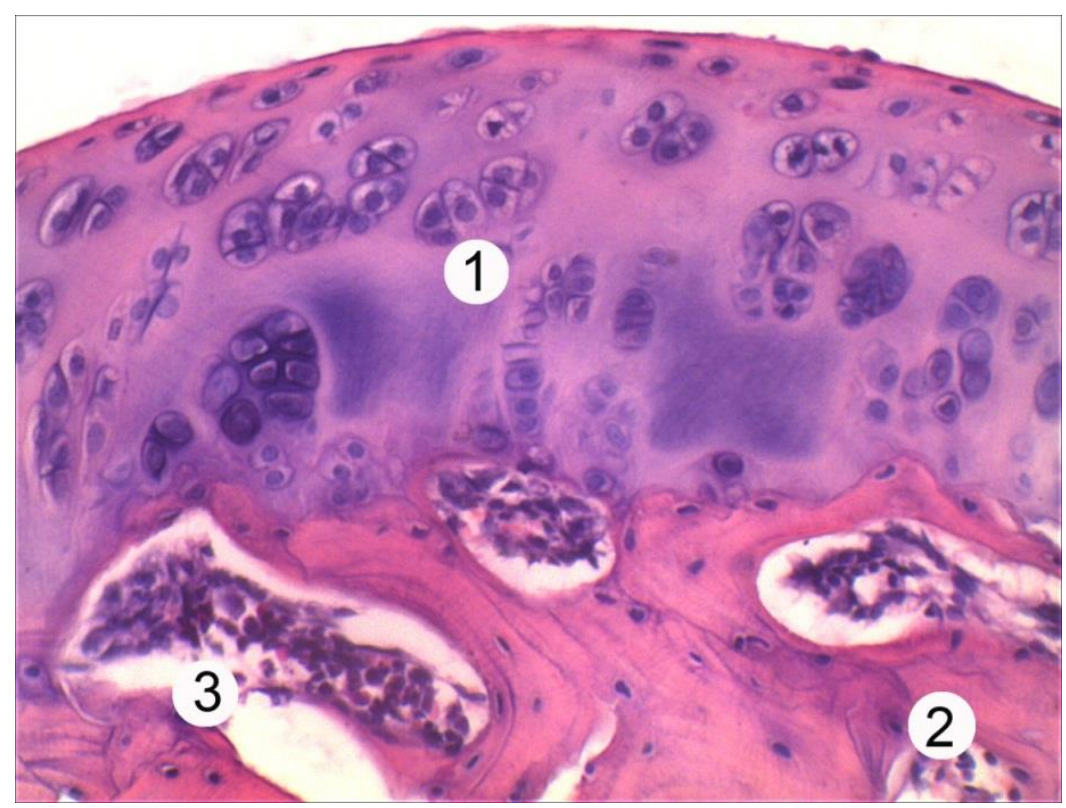

Figure 1. Histological changes in the hip joint of rats with experimental trauma. Articular cartilage (1), bone osteons (2), Haversian canal (3). Hematoxylin and eosin staining $\mathrm{x} 400$

In bone tissue, individual osteons look deformed, and their Haversian canals have a small area. In the spongy part of the bone, the bone beams look elegant, between them are wide cavities with red marrow. The fibrous layer of the joint capsule is swollen in places and does not fit tightly to the periosteum in the diaphysis (Fig. 2).

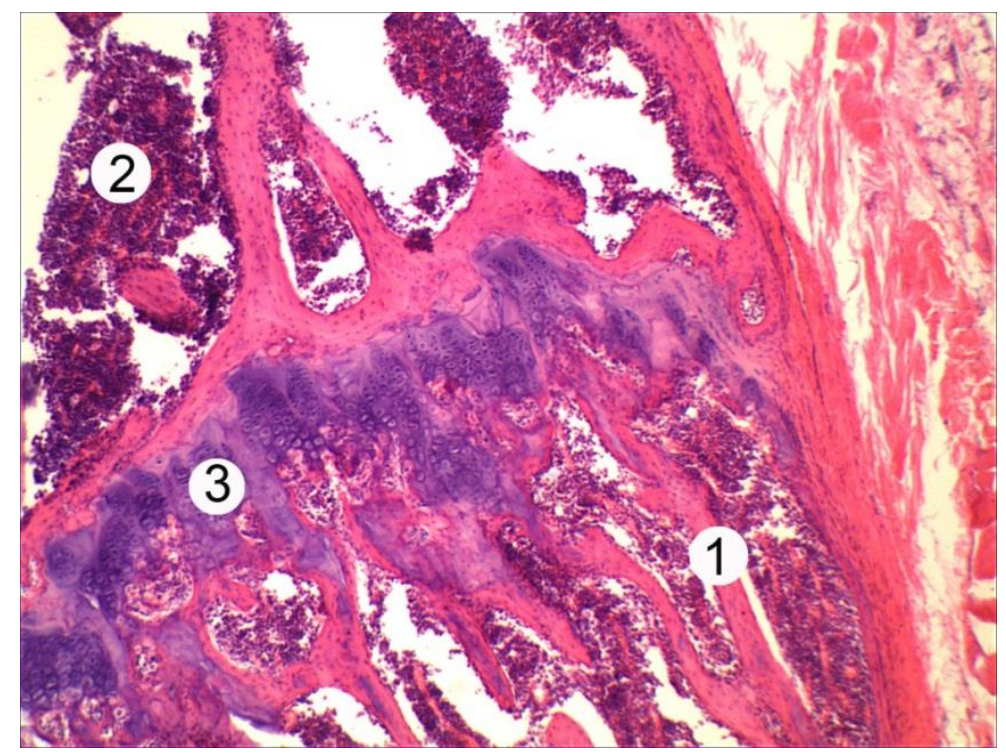

Figure 2. Histological changes in the hip joint of the animal with experimental trauma. Beams of the spongy part of the bone (1), the cavity with the red marrow (2), altered metaepiphyseal plate (3). Hematoxylin and eosin staining. x 100 
At high magnification of the optical microscope, significantly altered areas of hyaline cartilage of the metaepiphyseal plate of the joint are observed. The ordered arrangement of chondrocytes in the form of columns, a part of destructively changed cells, form accumulations, have pyknotic nuclei, and the increased basophilia of cytoplasm is lost. The intercellular substance in such areas becomes heterogeneous in color

Altered osteons and insertion plates are observed in the compact part of the hip bone. Tinctorial properties of the latter are violated, they are lighter in colour and lose the order of the plates, have the form of unstructured conglomerates (Fig. 3).

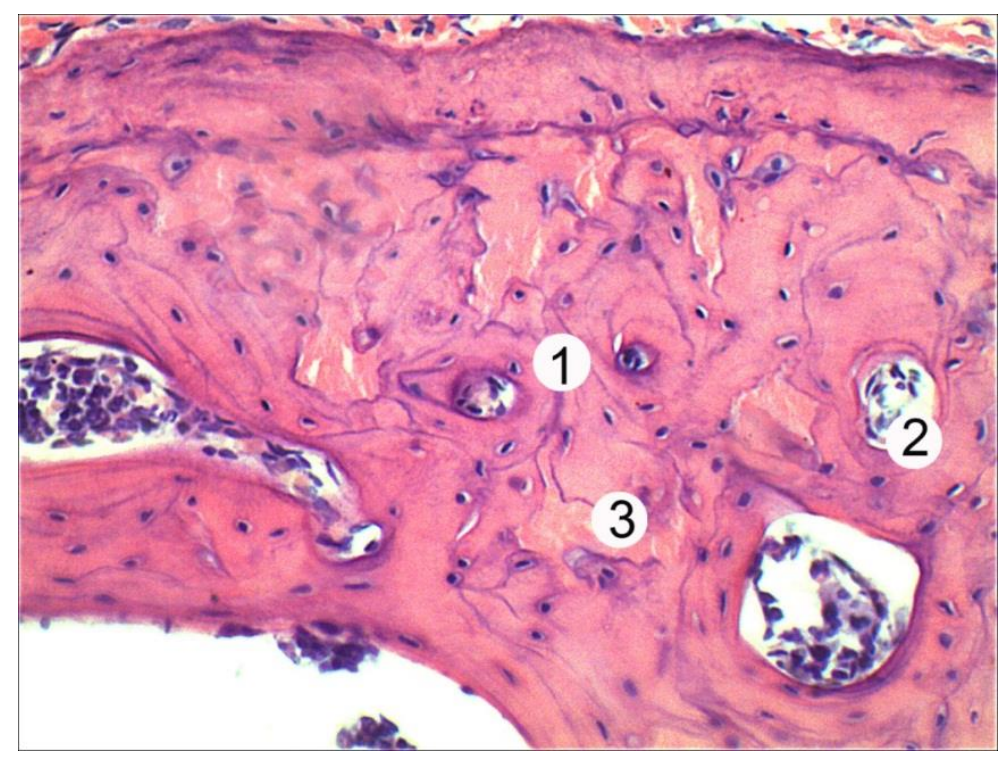

Figure 3. Histological changes in the hip joint of the animal with experimental trauma. Compact part of the bone (1), osteon (2), insert plates (3). Hematoxylin and eosin staining $\mathrm{x}$ 200

Microscopic examination of the knee joint of the animal in experimental trauma showed that the hyaline cartilaginous plate is slightly altered. There are some areas of increased basophilia, in which chondrocytes are damaged and their orderly arrangement is broken. The articular cavity is clearly limited by the articular capsule (Fig. 4).

Histologically, it was established that in the metaepiphyseal plate certain areas of the hyaline cartilage of the joint have changes in their organization.

There are few chondroblasts in its surface layer, so it is homogeneous, basophilic. There are also small cell-free zones throughout the thickness of the plate, where chondrocytes are solitary or absent (Fig. 5). 


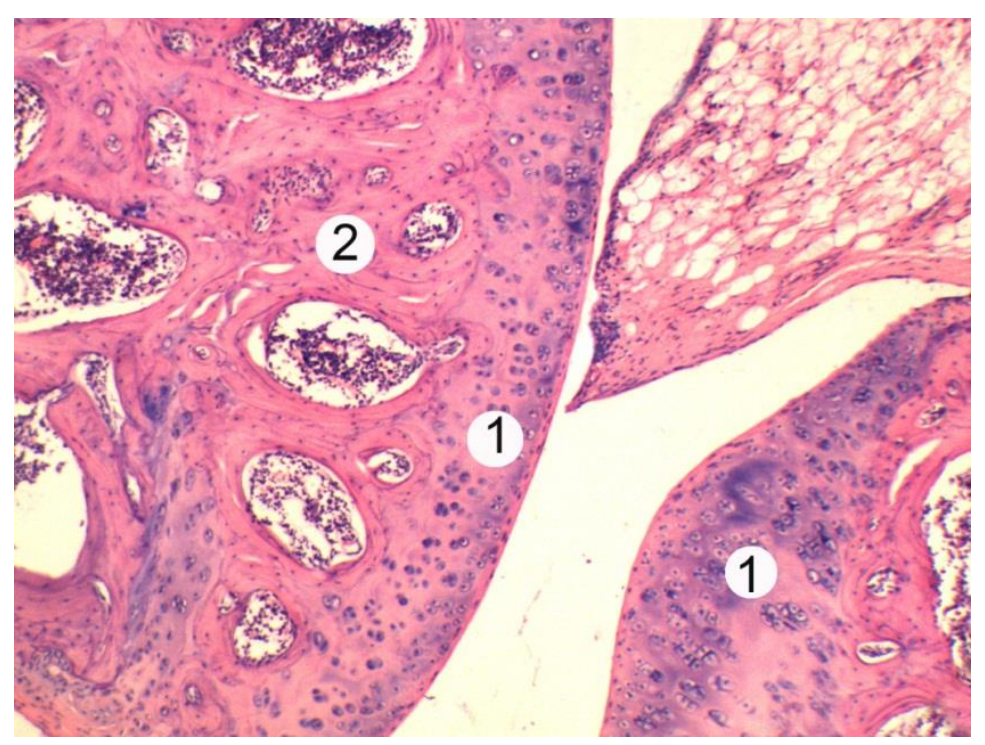

Figure 4 - Histological changes in the knee joint of the animal with experimental trauma. Superficial articular cartilage (1), bone tissue of the joint (2). Hematoxylin and eosin staining x 100

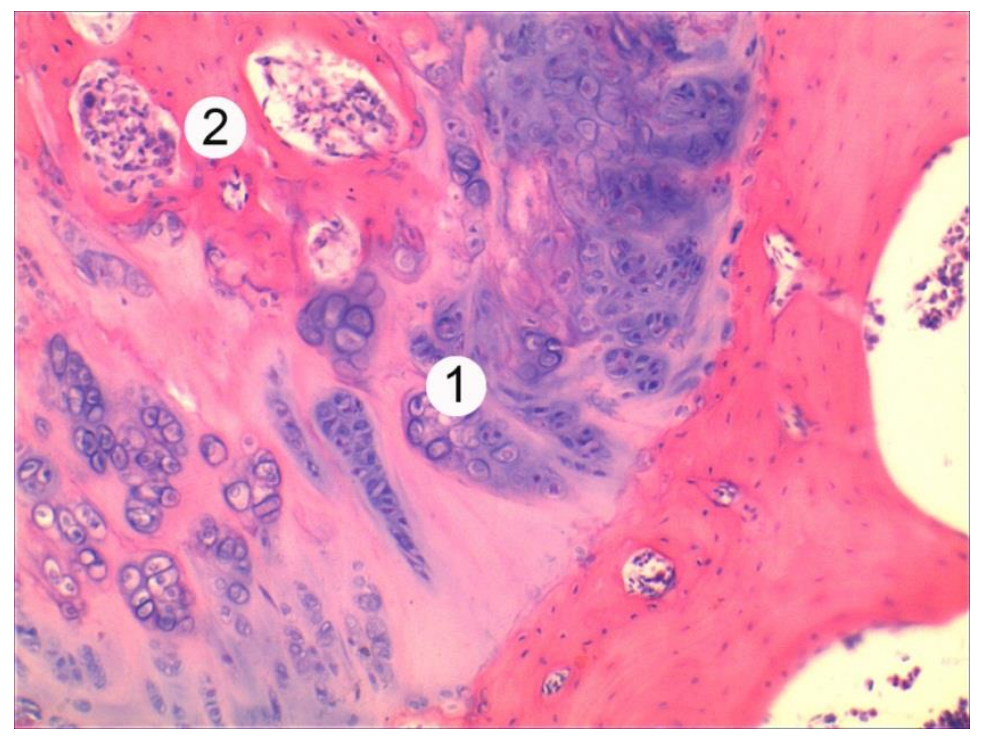

Figure 5. Histological changes in the knee joint of the animal with experimental trauma. Metaepiphyseal plate (1), spongy bone tissue (2). Hematoxylin and eosin staining. $\mathrm{x}$ 200

In the connective tissue surrounding the joint, there are blood-filled vessels, the wall of which has poorly defined membranes. There is no elastic membrane between the inner and middle shells. In loose connective tissue, there are small accumulations of histocytic infiltrate.

Therefore, the analysis of morphological changes after 21 days of experimental trauma revealed the presence in the hip and knee joints revealed reactive changes characteristic of 
inflammatory processes of large joints, namely: pronounced changes in hyaline cartilage of the metaepiphyseal plate of joints, connective tissue edema, significant vascular disorders in the form of arteries with a thickened wall and narrowed lumens, there was swelling of the adventitious lining of blood vessels.

\section{Conclusions}

1. Histological examinations have showed that 21 days after the simulation of the injury in the hip and knee joints there are reactive changes that are characteristic of inflammatory processes.

2. The established changes in vessels disturb a trophism of bones that causes changes of their structural components. The superficial articular cartilaginous plate responds to the injury simulation.

Prospects for further research. In the future, it is planned to develop pathogenetically sound means of correction of systemic changes that occur under the influence of limb reperfusion.

\section{References}

1. Trends in 1029 Trauma Deaths at a Level 1 Trauma Center/ T. Oyeniyi Blessing, BS, Erin E. Fox, Michelle Scerbo [et al.].// Injury. - 2017. - Vol.48(1). - P. 5-12.

2. The influence of fracture etiology and type on fracture healing: a review of 104 consecutive tibial shaft fractures / Karladani A., Granhed H., K?rrholm J. et al.// Archives of Orthopaedic and Trauma Surgery. - 2001 - Vol. 121 (6). - P.325-328.

3. Effect of repeated irrigation and debridement on fracture healing in an animal model/ Park S.-H., Silva M., Bahk W.-J. et al.// Journal of Orthopaedic Research. - 2002. Vol. 20(6). - P. 1197-1204.

4. Inflammation - a double edged sowrd in high-energy fractures?/ R. J. Bunn, G. Burke, C. Conelly et al.// The Bone \& Joint Journal. - 2005. - Vol. 87 (3). - P. 265-266.

5. Epidemiology of Posttraumatic Osteoarthritis /Abbey C. Thomas, Tricia HubbardTurner, Erik A. Wikstrom et al.// J Athl Train. - 2017. - Vol.52(6). - P. 491-496.

6. Johnson VL. The epidemiology of osteoarthritis/ V.L. Johnson, D.J. Hunter // Best Pract Res Clin Rheumatol. - 2014. - № 28. - P. 5- 15.

7. Managing of post-traumatic knee arthritis by total knee arthroplasty: case series of 15 patients and literature review/ Bahaa Ali Kornah, Hesham Mohamed Safwat, Said K. Abdel-hameed et al.// Journal of Orthopaedic Surgery and Research. -2019. - №.14. - P. 19. 
8. Osteochondral allograft transfer for treatment of osteochondral lesions of the talus: a systematic review./ VanTienderen RJ, Dunn JC, Kusnezov N, Orr JD. // Arthroscopy. 2017.- Vol.33(1). - P. 217-22. 